\title{
MULTI-WINDOWED SPECTRUM ESTIMATES OF THE ILS POLAR MOTION
}

\author{
L.A. Hinnov, U. S. Naval Observatory, Washington, D.C., USA \\ J. Park, Yale University, New Haven, Connecticut, USA
}

\begin{abstract}
We present a set of spectrum analysis techniques described by Thomson (1982) in which the discrete Fourier transform (DFT) is windowed by an expanding set of prolate spheroidal wave functions. The homogeneous ILS polar motion series was chosen for the presentation because of its interesting spectrum. It contains lines at the annual and semi-annual frequencies, a band of uncertain width and structure around Chandler's frequency, and evidence for a 30year periodicity. None has been measured with great certainty by traditional single-taper DFT spectrum analysis. Here, a multi-taper algorithm significantly improves the bias control and consistency of high-resolution DFT spectrum estimates. An analysis of variance is performed to locate significant harmonic lines, and jackknifed statistics are used as additional cross-checks on the stability of the results. In addition, multi-tapered coherence estimates confirm a previously noted correlation between a meteorological forcing function and a polar motion excitation derived from the ILS series.
\end{abstract}

\section{INTRODUCTION}

Five components have been identified in the ILS polar motion series: (1) a dominant 14-month Chandler wobble, (2) a forced annual wobble, (3) a slowly varying secular drift, (4) a 30-year oscillation, and with lesser certainty, (5) a semi-annual variation. A central controversy concerns a line separation observed within the Chandler resonance band near $+0.835 \mathrm{cpy}$ and $+0.853 \mathrm{cpy}$. Federov \& Yatskiv (1965) suggested that this "bifurcation" was the product of a phase reversal in the data that took place from 1924-1926 due to changes in the ILS star catalogue. However, McCarthy (1974) discovered a similar split peak in the periodogram of an independent set of latitude observations taken at Washington, D.C., for which the star catalogue has never been modified. This suggested that the double peak was not an artifact of an ILS observing error, but evidence of an excitation that was causing the (single-component) Chandler resonance to modulate. Okubo (1982) analyzed synthetic single Chandler frequency polar motion sequences to demonstrate apparent Chandler frequency fluctuations for $50<Q_{W}<100$, thus ending speculation that the phenomenon evidenced a multicomponent process.

Here we reaffirm the existence of the two apparent lines near Chandler's frequency using the spectrum estimation method developed by Thomson (1982). In addition, we analyze lines at seasonal frequencies, and within the low frequency band near the 30-year component. The ILS series is tapered by a set of ordered 
prolate spheroidal sequences (Slepian 1978). Each tapered version of the series is then Fourier transformed separately by DFT, and the resulting collection of Fourier transforms is used as a set of doubly orthogonal estimators of the true spectrum. An analysis of variance is performed on these estimators to locate statistically significant harmonic lines, with "jackknifed" statistics (Efron 1982) supporting the reliability of the results.

This multi-windowing is particularly useful in the study of the Chandler wobble's excitation, in which averaged coherence spectra are formed using the multi-windowed Fourier transforms of hypothetical and observed excitation series. The ability of the technique to maximize statistical stability while maintaining the extremely narrow band required to resolve the Chandler band cannot be duplicated by single-taper methods.

\section{MULTI-TAPER SPECTRUM ANALYSIS}

The procedures applied to the ILS series below are discussed at great length by Thomson (1982). The basic algorithm involves a total of K DFTs of the complex, time-sampled ILS series $x_{n}$ of length $N$, tapered by functions $v_{k, n}\left(N, f_{w}\right)$ for $k=$ $0,1, \ldots, \mathrm{K}-\mathrm{l}$ :

$$
y_{k}(f)=\sum_{n=0}^{N-1} x_{n} v_{k, n} \exp [-i 2 \pi f(n-(N-1) / 2)]
$$

The $v_{k, n}$ are the first $K$ of $N$ ordered eigenvectors satisfying a system of $N$ lagged discrete Dirichlet kernels of resolution $2 \mathrm{Nf}_{\mathrm{w}}, 0<\mathrm{f}_{\mathrm{w}}<\mathrm{f}_{\text {nyq }}$, formally known as "discrete prolate spheroidal sequences" (Slepian 1978). "Their eigenfunction derivation has since earned them the name "eigentapers"; the $y_{k}(f)$ in (1) are "eigencoefficients"; and $\hat{S}_{k}(f)=\left|y_{k}(f)\right|^{2}$ is an "eigenspectrum" of order $k$ of the data. Their associated ordered eigenvalues $L_{k}$ provide measures of the fraction of the total energy of the spectrum preserved within the main lobe of the spectral window of their associated $v_{k, n}$, i.e., the "discrete prolate spheroidal wave function," or "eigenwindow" $\mathrm{U}_{\mathrm{k}}(\mathrm{f})$. The first $2 \mathrm{Nf}_{\mathrm{w}}$ eigenvalues are extremely close to unity, after which they rapidly drop off to zero; thus, only the first $\mathrm{K}=$ $2 \mathrm{Nf}_{\mathrm{w}}$ ordered eigentapers are utilized in the algorithm. The bias of the kth eigenspectrum is approximated by $B_{k}(f)=\left(1-L_{k}\right) s^{2}$, where $s^{2}$ is the sample variance of the data.

The best estimate of the true spectrum $S(f)$ is a weighted sum of the $K$ eigenspectra, or the "adaptive-weighted spectrum" with a half-bandwidth resolution $\mathrm{f}_{\mathrm{w}}$ :

$$
\hat{S}(f)=\sum_{k=0}^{K-1}\left|d_{k}(f)\right|^{2} \hat{S}_{k}(f) / \sum_{k=0}^{K-1}\left|d_{k}(f)\right|^{2}
$$

where the adaptive weights $d_{k}(f)$ are estimated statistically by minimizing the least squares "misfit" between the estimated spectrum and the unknown true spectrum, incorporating estimates of the broadband leakage bias $B_{k}(f)$. The effective number of degrees of freedom (d.f.s) for (2) is given by:

$$
u(f)=2 \sum_{k=0}^{k-1}\left[L_{k}\left(d_{k}(f)\right)^{2} / L_{0}\left(d_{0}(f)\right)^{2}\right]
$$

To estimate harmonic lines at local frequencies, a "point regression" technique was devised, based on the assumption that estimated mean values $\hat{m}(f)$ of the $K$ adaptive-weighted eigencoefficients are non-zero at frequencies containing 
lines. The square of such an estimated mean is an alternative measure of (2). Subtracting $\hat{m}(f)$ from the $K$ eigencoefficients produces a residual which, when squared, becomes an estimate of the local background (noise) spectrum. The ratio of these two quantities is equivalent to an F-statistic with 2 and $u(f)-2$ d.f.s, and is a direct signal-to-noise ratio (SNR) estimate of the local spectrum:

$$
F(f)=\frac{\left(y_{2 u} u(f)-1\right)|\hat{m}(f)|^{2} \sum_{k=0}^{k-1} U_{k}(0)^{2}}{\left|\sum_{k=0}^{k-1} d_{k}(f) y_{k}(f)-\hat{m}(f) u_{k}(0)\right|^{2}}
$$

$F(f)$ is used as $a=0$ test for the significance of suspected harmonic spectral components.

The statistical "jackknife" is performed by computing (4) $\mathrm{K}$ times, each time withholding one of the $\mathrm{K}$ eigencoefficients. These jackknifed estimators are used to infer additional statistics. For example, their average is also an estimate of the SNR, with a standard deviation (s.d.) indicating whether all $\mathrm{K}$ eigenwindows have sampled a significant cyclicity (s.d. small), or whether a single eigencoefficient has biased the average with an inordinately large value not present in the other $\mathrm{K}-1$ eigencoefficients (s.d. large). We average $\log (\mathrm{F})$ rather than $F$, as its distribution is less prone to bias from extreme values.

\section{ILS "EIGENESTIMATES"}

The adaptive-weighted spectrum in Figure 1 was computed from a trendless, zeromean version of ILS data, and was interpolated by zero padding the data to four times its original length prior to the DFT. High amplitude spectra are concentrated in the lower frequencies, with strong peaks centered on \pm 1.0 cpy and +0.843 cpy. The peak near 0 cpy contains the residual variances of partial frequencies lower than the Rayleigh spacing of 1/80 cpy. The half-bandwidth resolution was selected at $f_{w}=0.05 \mathrm{cpy}$, to ensure that the Chandler band, which has an unknown width, was sampled in its entirety. For the 80 -year series length, this results in a set of $2 \mathrm{Nf}_{\mathrm{w}}=8$ eigenspectra. The uniform broadness of the peaks is due to the effective eigenwindow that results from combining the eight eigenspectra. The optimizing effect of the computed adaptive weights $d_{k}(f)$ yields in high estimated stability in regions with non-zero variance.

Results of the analysis of variance appear in Table 1. Significance was determined by integrating F-distributions with 2 and $u(f)-2$ d.f.s up to the indicated estimated values. To isolate these spectral lines at these levels of significance, it was necessary to zero pad the input eigentapered series up to 8 times the original series length. Two peaks emerge from the Chandler band, corresponding to 437-day and 428-day periods. Their estimated values are lower than one might expect; according to Dickman (1981) this is due to greater noise in this part of the spectrum, originating from errors in ILS astrometry. The annual frequency has a prograde $(+1.0$ cpy) F-statistic exceeding the $99.99 \%$ significance level, evidencing steady oscillation. The retrograde annual component is slightly lower, due to its greater spectral distance from the amplifying influence of the Chandler resonance. The line at the semi-annual frequency is less significant, with a prograde component at $88 \%$. The only other known measurements of the ILS semi-annual term are by Rudnick (1956) and Walker \& Young (1957), for which the uncertainties are on the order of the estimated values. The analysis of variance near 0 cpy produces a single peak at the 32-year period, at -0.031250 cpy, exceeding $99 \%$ significance. The estimated SNR of 9.72 is considerably less 

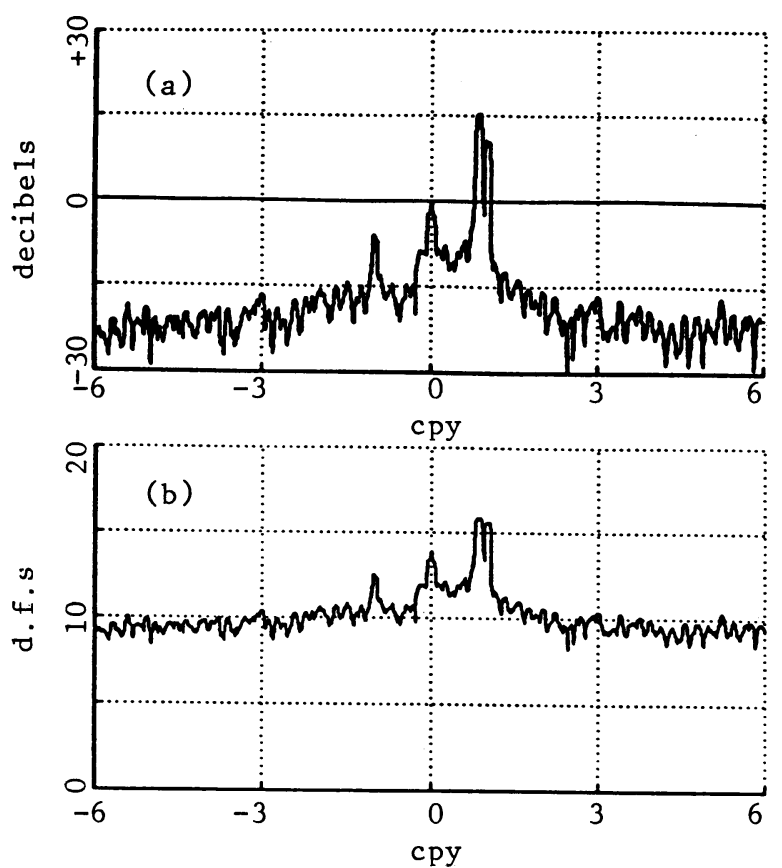

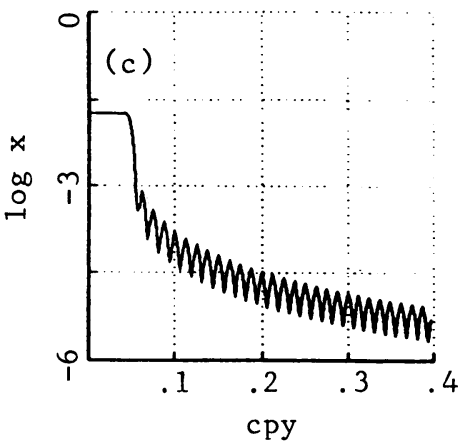

Figure 1. (a) The adaptive-weighted sum of eight eigenspectra across the ILS Nyquist range, with a bandwidth resolution of $0.1 \mathrm{cpy}$, computed from 80 years of monthly mean ILS observations, from January 1900 to December 1979. (b) The estimated stability of this spectrum, in degrees of freedom. The maximum recoverable d.f.s is 16 over the $0.1 \mathrm{cpy}$ bandwidth. (c) The effective eigenwindow of this spectrum, which is the average of eigenwindows $U_{k}(f)$ for $k=0$ through 7 .

TABLE 1. F-distribution probability of jackknifed estimators at selected frequencies.

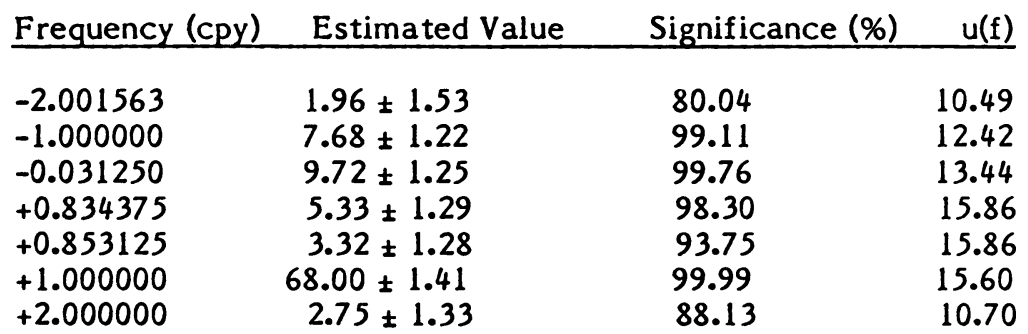


than the 21 to 1 estimate by Dickman (1981), who used least squares fits of the data to 31-year period sinusoids plus a constant, but nonetheless establishes the ability of the multi-tapering to optimize DFT spectrum estimation over extremely narrow low frequency bands.

The multi-taper algorithm can be applied in the study of the polar motion's excitation. The excitation of the Chandler wobble must be examined using as long a data series as possible, and this makes the ILS series the first choice for analysis. Hinnov \& Wilson (1986) show that a significant coherence peak occurs in the Chandler band between an excitation derived from 70 years of ILS observations and an excitation covering the same time period calculated from meteorological phenomena. Figure 2 compares their coherence-squared spectrum, which was estimated using a single $20 \%$ Tukey window, to the coherence-squared spectrum formed by eigencoefficients estimated from the same excitations. The chief difference between the two coherence estimates is that the Tukey-windowed version contains only 12.0 degrees of freedom, whereas the eigenwindowed version has recovered 13.74, or nearly all of the 14 degrees of freedom theoretically available within the 0.1 cpy averaging bandwidth. Such a difference can be critical for the successful detection of a true correlation within a narrow, sparsely sampled band embedded in a noisy spectrum. Therefore, the algorithm is highly recommended for investigators of the polar motion's excitation.

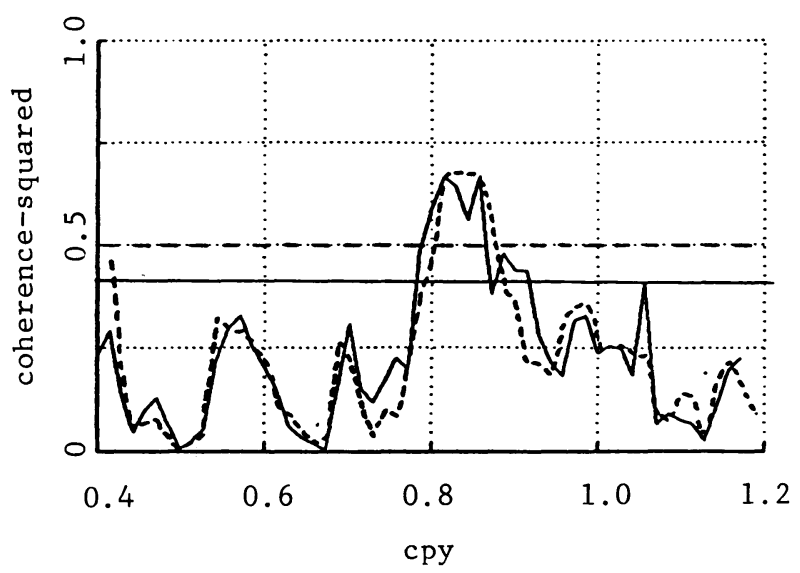

Figure 2. Coherence-squared spectrum estimates between the ILS and meteorological excitations of Hinnov \& Wilson (1986). The dashed curve shows estimates of the spectrum using a $20 \%$ Tukey window, averaged over 7 adjacent Rayleigh spacings (equal to a 0.1 cpy bandwidth resolution) for a total of 12.0 d.f.s. The $95 \%$ confidence for zero coherence is given by the horizontal dashed line. The solid curve is the same spectrum estimated by eigencoefficients $y_{k}(f)$ of the two excitations, also with a 0.1 cpy resolution, but with 13.74 d.f.s. The $95 \%$ confidence level for this spectrum is given by the horizontal solid line. 


\section{DISCUSSION}

This study demonstrates the extent to which the multi-taper algorithm compensates for the spectral leakage and inconsistency of DFT spectrum estimates of the ILS series across exceptionally narrow bands. In addition, the eigenspectra provide excellent regressors for line component identification near the Rayleigh spacing to significance levels unattainable by single-taper DFT analysis. For example, a "mild" $20 \%$ Tukey-windowed ILS spectrum reduces the d.f.s over a 0.1 cpy bandwidth of the 80-year monthly ILS spectrum from a total possible of 16 to 13.6, and provides poor leakage resistance (see Park et al. 1986 for details). Single tapers with better leakage resistance result in even further reductions in d.f.s because they invariably require a heavy suppression of sampled values near the extremities of the data series. In contrast, the adaptive-weighting procedure recovers nearly all of the 16 total degrees of freedom available in the 0.1 cpy smoothing bandwidth around the Chandler frequency in the discrete ILS spectrum.

These procedures have confirmed each of the known components in the ILS spectrum with well constrained estimates derived from an eigenfunction expansion of the DFT. The ILS semi-annual variation, for example, was detected to a level of significance not previously achieved. With higher quality polar motion data now available, the multi-taper algorithm may also prove useful in constraining estimates of very high frequency variations. Also within the next year, the controversial spectral components, i.e., the Chandler bifurcation and the 30-year term, can be investigated by applying these techniques to the independently observed BIH polar motion. By the close of 1986 this data series will yield a Rayleigh spacing of 1/30 cpy, which is on the order of the line separation of the Chandler bifurcation. The analysis of variance, which has been seen to resolve lines at the Rayleigh spacing, conceivably could detect both the bifurcation and a 30-year cycle, if they are indeed present.

\section{REFERENCES}

Dickman, S.R.: 1981, J.Geophys. Res., 86, 4904.

Efron, B.: 1982, SIAM, 1 .

Federov, E.P and Ya. S. Yatskiv: 1965, Soviet Astron., 8, 608.

Hinnov, L.A. and C.R. Wilson: 1986 , submitted to Geophys. J. R. astr. Soc.

McCarthy, D.D.: 1974, J. Geophys. Res., 79, 3343.

Okubo, S.: 1982, Geophys. J. R. astr. Soc., 629.

Park, J., Vernon III, F.L., and C.R. Lindberg: 1986, submitted to

J. Geophys. Res.

Rudnick, P.: 1956, AGU Trans., 37, 137.

Slepian, D.: 1978, Bell Systems Tech. J., 57, 1371.

Thomson, D.J.: 1982, IEEE Proc., 70, 1055.

Walker, A., and A. Young: 1957, Mon. Not. R. astr. Soc., 117, 119.

Yumi, S., and K. Yokoyama: 1980, Publ. Centr. Bureau I.P.M.S., Mizusawa.

\section{DISCUSSION}

Chao: What is your criterion of choosing the number of eigentapers?

Reply by Hinnov: We choose those eigentapers whose associated eigenvalues $\approx 1$. In the case of the ILS series of length $\mathbf{N}=80$ years and a spectrum to be expanded over $2 \mathrm{~W}=0.1 \mathrm{cpy}$, this results in $2 \mathrm{NW}=8$ eigentapers. For further discussion, we refer you to Thomson (1982). 